\section{Interação entre vitamina A e ferro em diferentes grupos populacionais}

\section{Vitamin A and Iron interaction in different populations}

Michele Pereira Netto 1

Silvia Eloiza Priore 2

Sylvia do Carmo Castro Franceschini 3

\begin{abstract}
Interaction between iron and vitamin A has been the focus of scientific research for the reason that some of the issues still remain unknown. A literature review through Medline of the National Library of Medicine, United States of America, database selected scientific publications addressing interaction of iron and vitamin A in humans beginning in the 80's and proceeding to the year of 2003. The key words used in the literature review were "iron", "vitamin A" and "anemia" and corresponding terminology in Portuguese. Results indicates a correlation between vitamin A and iron nutritional indicators in children, adolescents, and pregnant women establishing also that vitamin A fortification and supplementation has a favorable effect on the iron metabolism of these populations. Further studies are required to establish dosages and time of vitamin A supplementation necessary to significantly benefit the iron nutritional status. Mechanisms explaining the association of vitamin A and iron are still ignored, however it is the understanding of some authors that this interaction results from the fact that vitamin A benefits erythropoiesis, prevents infections and improves iron absorption.
\end{abstract}

Key words Iron, Vitamin A, Anemia

\section{Resumo}

A interação entre ferro e vitamina A tem sido alvo de pesquisas científicas, visto que existem questões ainda pouco esclarecidas. Através de um levantamento bibliográfico, mediante consulta à base de dados Medline da National Library of Medicine, Estados Unidos da America, foram selecionadas publicações que tratavam sobre a interação entre ferro e vitamina $\mathrm{A}$ em seres humanos, a partir da década de 80 até o ano de 2003. As palavras chave utilizadas no levantamento bibliográfico foram "ferro", "vitamina A" e "anemia" e seus correspondentes em língua inglesa. Os resultados indicam que existe correlação entre os indicadores do estado nutricional de vitamina A e ferro em crianças, adolescentes $e$ gestantes e que a fortificação e suplementação com vitamina A têm efeito favorável no metabolismo do ferro nesses grupos. Entretanto, ainda são necessários mais estudos para se conseguir estabelecer quais as doses e o período necessário de suplementação de vitamina A para beneficiar significantemente o estado nutricional de ferro. Os mecanismos que explicam a associação entre vitamina A e ferro ainda são desconhecidos, entretanto alguns autores atribuem essa interação ao fato de a vitamina A beneficiar a eritropoese, prevenir infecções ou melhorar a absorção de ferro.

Palavras-chave Ferro, Vitamina A, Anemia 


\section{Introdução}

O ferro apresenta várias funções vitais para o organismo, servindo como carregador de oxigênio através da molécula de hemoglobina, como meio de transporte de elétrons para dentro das células e como parte integrante de importantes sistemas enzimáticos em vários tecidos. ${ }^{1}$ A deficiência desse mineral pode acarretar agravos à saúde, tais como, alterações da capacidade de trabalho físico e da atividade motora, alterações da imunidade celular e da capacidade bactericida dos neutrófilos, maior susceptibilidade às infecções, diminuição da termogênese, alterações funcionais e histológicas do tubo digestivo, comprometimento da mobilização hepática da vitamina $\mathrm{A}$, maior risco de parto prematuro e de morbidade perinatal, menor transferência de ferro ao feto, diminuição da velocidade de crescimento e redução da capacidade cognitiva.1-6 Algumas dessas alterações são próprias da anemia e outras causadas pelo mal funcionamento de enzimas dependentes de ferro. ${ }^{2}$

A vitamina A é um nutriente essencial, importante na promoção do crescimento e desenvolvimento, e ainda na manutenção da integridade epitelial, função imune e reprodução. ${ }^{7}$ A deficiência de vitamina A está associada com um aumento acentuado da morbi-mortalidade, principalmente por doenças infecciosas, e a deficiência grave pode levar a problemas de visão que chegam até a cegueira total. 8

A concomitante ocorrência de anemia e deficiência de vitamina A foi estudada em crianças de um a cinco anos em Honduras, e encontrou-se entre as crianças de 12 a 36 meses prevalência de $21,6 \%$ e nas maiores 9,3\%.9 Pré-escolares estudados por Palafox et al.10 apresentaram as seguintes prevalências de deficiência de vitamina A, deficiência de ferro, anemia e deficiência conjunta de vitamina A e ferro: $59,9 \% ; 53,5 \% ; 36,4 \%$ e $33,2 \%$, respectivamente. Além disso, o risco de lactentes e lactantes com deficiência de vitamina A apresentarem anemia foi 2,9 e 3,8 vezes maior quando comparado com aquele observado nesses grupos biológicos sem deficiência. ${ }^{11}$ A elevada co-ocorrência das duas deficiências demonstra a necessidade de intervenções nutricionais em conjunto.

A associação entre anemia ferropriva e hipovitaminose A tem sido estudada, entretanto os mecanismos dessa associação não estão bem esclarecidos. Acredita-se que a vitamina A influencie na anemia via modulação da hematopoiese, por melhorar a imunidade para doenças infecciosas 12,13 e através da modulação do metabolismo de ferro. 12

O propósito deste trabalho foi revisar os estudos existentes sobre a interação entre ferro e vitamina A em crianças, adolescentes, gestantes e mulheres em idade fértil.

\section{Métodos}

Foi feito um levantamento bibliográfico mediante consulta às bases de dados Medline da National Library of Medicine, Estados Unidos da América, tendo sido selecionadas as publicações que tratavam da interação entre ferro e vitamina A em seres humanos, a partir de 1980, até 2003. As palavras chave utilizadas no levantamento bibliográfico foram "ferro", "vitamina A" e "anemia" e seus correspondentes na língua inglesa.

\section{Correlação entre o estado nutricional de ferro e vitamina A}

Muitos estudos têm mostrado a correlação entre os indicadores do estado nutricional de ferro e vitamina A entre crianças, 14-19 gestantes 20,21 e adolescentes. $22-24$

Em crianças tailandesas, de um a oito anos, foram encontradas correlações fracas, entretanto significantes, entre níveis de retinol e hematócrito, ferro sérico, ferritina e porcentagem de saturação de transferrina; RBP (proteína de ligação do retinol) e hemoglobina, hematócrito, ferro sérico, ferritina, e porcentagem de saturação de transferrina; encontrou-se ainda correlação negativa entre transferrina com retinol e RBP. 15 A correlação entre a hemoglobina e o retinol plasmático, em pré-escolares da Etiópia também foi significante.16 Entre pré-escolares mexicanos anêmicos 17 houve correlação entre hemoglobina e retinol e hematócrito e retinol. Em pré-escolares brasileiros, também se observou correlação entre hemoglobina e retinol, embora a ferritina analisada não se tenha mostrado associada com o retinol plasmático. ${ }^{18} \mathrm{Em}$ estudo recente com crianças de nove meses de idade que não apresentavam sinais de deficiência de vitamina A, observouse correlação positiva entre retinol sérico e concentração de hemoglobina e ferro sérico; entretanto não foi verificada associação com a ferritina. 19

A interação entre vitamina A e ferro em adolescentes tem sido pouco estudada. O primeiro estudo nesse grupo foi conduzido em Bangladesh com meninas entre 12 e 15 anos, 22 no qual foram observadas correlações entre retinol sérico e hemoglobina, ferro sérico e percentual de saturação da transferrina. Em outro estudo com adolescentes do sexo feminino, 
com idade entre 10 e 19 anos, observou-se correlação entre hemoglobina e retinol sérico. ${ }^{23} \mathrm{Em}$ adolescentes anêmicas, de 14 a 19 anos, encontrouse correlação entre retinol sérico e hemoglobina, mas a correlação entre retinol e ferritina esteve apenas no limite da significância $(\mathrm{r}=0,11 ; p=0,06){ }^{24}$

As correlações entre os indicadores do estado nutricional de ferro e vitamina A também foram estudados em gestantes. Na Indonésia, o retinol sérico de gestantes esteve positiva e significantemente associado com hemoglobina, hematócrito e ferro sérico. Para essa avaliação, utilizou-se a análise de regressão multivariada para ajustar os efeitos da idade gestacional, paridade e local de moradia das gestantes, e observou-se que cada aumento de $1 \mu$ $\mathrm{mol} / \mathrm{L}$ na concentração de retinol esteve associado com aumento de 4,3 g/dL de hemoglobina, $1,37 \%$ de hematócrito e $1,18 \mu \mathrm{mol} / \mathrm{L}$ de ferro sérico. $21 \mathrm{Na}$ Tanzânia, gestantes foram dividas em três grupos de acordo com a concentração de hemoglobina: maior ou igual a $11 \mathrm{~g} / \mathrm{dL}$, entre 9 e $10,9 \mathrm{~g} / \mathrm{dL}$ e menor que 9 $\mathrm{g} / \mathrm{dL}$, observando-se que as concentrações de retinol sérico diminuíram significantemente com a redução de hemoglobina $(0,93 ; 0,84$ e $0,75 \mu \mathrm{mol} / \mathrm{L}$; respectivamente); ou seja, baixas concentrações de vitamina A estavam associadas à baixas concentrações de hemoglobina. 20

Em nutrizes do Kenia que amamentaram por mais de quatro meses se observou correlação entre retinol e hemoglobina e retinol e ferritina. 25

Os resultados apresentados demonstram que, embora fracas, existem associações entre os indicadores do estado nutricional de vitamina A e ferro em crianças, adolescentes e gestantes. Entretanto, esses resultados devem ser analisados com cautela, pois trata-se de estudos transversais ou de dados iniciais de diagnóstico de estudos de suplementação e, portanto, não se pode inferir uma relação causaefeito das variáveis estudadas.

\section{Efeito da suplementação com vitamina A no estado nutricional de ferro}

O efeito benéfico da suplementação e fortificação da vitamina A no metabolismo do ferro tem sido comprovado em diferentes grupos populacionais. ${ }^{15,24,26-33}$

As crianças são o grupo populacional mais estudado para verificar a interação entre vitamina A e ferro. A eficácia de um programa de fortificação do açúcar com vitamina A no metabolismo do ferro de crianças de um a cinco anos residentes na Guatemala foi estudada e, após seis meses de fortificação observou-se aumento na capacidade total de ligação do ferro (CTLF) e redução da ferritina. Contudo, após dois anos de fortificação, todos os indicadores do estado nutricional desse mineral melhoraram: ferro sérico, percentual de saturação da transferrina e ferritina aumentaram e a CTLF reduziu-se significantemente. A interpretação dos autores é que a vitamina A aumenta a biodisponibilidade do ferro sérico para hematopoiese devido à depleção dos estoques, provocando uma melhora na absorção do ferro dietético, e a longo prazo, eleva os níveis de ferritina e reduz a CTLF. ${ }^{26}$ Com relação a esse estudo26 é necessário ressaltar que, na Guatemala, embora não descritas pelos autores, as prevalências de hipovitaminose A e deficiência de ferro são elevadas. Outro estudo na Guatemala, em crianças anêmicas de um a oito anos suplementadas com $3 \mathrm{mg}$ de vitamina A isolada ou em combinação com o ferro durante dois meses, mostrou elevação nos níveis de hemoglobina, hematócrito, eritrócitos, ferro sérico e percentual de saturação da transferrina, não tendo entretanto, efeito sobre a capacidade total de ligação de ferro e ferritina sérica. A elevação do ferro sérico foi maior quando a vitamina A e ferro, em conjunto, foram administrados. Durante o estudo, os grupos de suplementação foram randomizados e, portanto, as médias de retinol e hemoglobina na baseline não apresentavam diferenças estatísticas entre os grupos estudados. Os autores concluíram que o efeito primário da suplementação de vitamina A é o incremento dos níveis de ferro sérico. 27

Em 1989, crianças tailandesas anêmicas de um a seis anos, após dois meses recebendo cápsulas com $110 \mathrm{mg}$ de vitamina A, tiveram um aumento significante nas médias de ferro sérico e percentual de saturação da transferrina, comparadas ao grupo controle que não recebia nenhum tipo de suplementação. 15 No ano seguinte, o mesmo grupo de pesquisadores 28 analisou o efeito da suplementação com $110 \mathrm{mg}$ de vitamina A durante um período de duas semanas em crianças de três a nove anos, deficientes em vitamina A, e observaram depois da intervenção que as concentrações de hemoglobina, hematócrito, ferro sérico e saturação da transferrina aumentaram significantemente; entretanto ferritina e transferrina não sofreram alterações. Os autores concluíram que a melhora no metabolismo do ferro com a suplementação de vitamina A provavelmente não é resultado do impacto direto da absorção de ferro, mas da mobilização do ferro armazenado e do aumento da utilização desse ferro para formação da hemoglobina; os estoques de ferro diminuem provocando um aumento na absorção desse mineral. Nos dois estudos citados anteriormente 15,28 os dados do 
estado nutricional de ferro e vitamina A e os antropométricos não diferiam entre os grupos de suplementação e controle.

$\mathrm{Na}$ Tanzânia, 136 crianças pré-escolares anêmicas sem sinais de deficiência de vitamina $A$ receberam 1,5 mg de vitamina A durante três meses. Após esse período observou-se melhora significante na concentração de hemoglobina nos grupos que receberam ferro, vitamina A e vitamina A mais ferro, sendo que este último esquema de suplementação teve melhor resultado com maiores ganhos nas médias de hemoglobina e menores prevalências de anemia. No período inicial do estudo não houve diferença estatística entre as características demográficas e nutricionais dos grupos. ${ }^{29}$

Em estudo do tipo randomizado, duplo-cego, placebo-controlado, com adolescentes anêmicas de 14 a 19 anos, de Bangladesh, sem deficiência de vitamina A, foi feito suplementação semanalmente, durante 12 semanas, de ferro, ferro mais ácido fólico, ou ferro adicionado de ácido fólico mais vitamina A. Após esse período, observaram-se aumentos significantes na concentração de hemoglobina: 0,33 ; 0,91 e $1,22 \mathrm{~g} / \mathrm{dL}$ para os grupos que receberam vitamina A $(2,42 \mathrm{mg})$, ferro mais ácido fólico e ferro, ácido fólico e vitamina $\mathrm{A}$, respectivamente. Em consequiência do aumento da concentração de hemoglobina observou-se a redução da prevalência de anemia depois dos tratamentos de 27,52 e $63 \%$ nos três grupos, respectivamente. 24

Também tem sido estudada a importância da suplementação de vitamina A no estado nutricional de ferro de gestantes. Em gestantes indianas, observou-se aumento significante na concentração de hemoglobina quando essas foram suplementadas com ferro mais $1,8 \mathrm{mg}$ de vitamina A durante um período superior a 12 semanas e, esse aumento foi significantemente maior nesse grupo comparado àquele que era suplementado apenas com ferro. ${ }^{31}$

A suplementação com $2,4 \mathrm{mg}$ de vitamina A durante oito semanas em gestantes anêmicas da Indonésia produziu um incremento na concentração de hemoglobina de 0,$37 ; 0,77$ e $1,28 \mathrm{~g} / \mathrm{dL}$ nos grupos que receberam vitamina $\mathrm{A}$, ferro e vitamina $\mathrm{A}$ mais ferro; respectivamente. A alteração no ferro sérico e percentual de saturação da transferrina depois da suplementação acompanharam a mesma tendência da concentração de hemoglobina. A recuperação da anemia foi de $35 \%, 68 \%$ e $97 \%$ nos grupos que receberam vitamina $\mathrm{A}$, ferro e ambos; respectivamente. Vale ressaltar que a população de gestantes avaliadas não apresentava deficiência de vitamina $\mathrm{A}$, visto que a média do retinol plasmático na baseline era de 1,08 $\mu \mathrm{mol} / \mathrm{L} .30$
A suplementação semanal, durante 17 semanas, de $3 \mathrm{~g}$ de vitamina A em gestantes da Indonésia ${ }^{33}$ aumentou a concentração de hemoglobina e reduziu a de ferritina, diferente da suplementação com ferro apenas, na qual não se observou alteração significante nos dois parâmetros; com esses resultados os autores postularam que as alterações na hemoglobina e ferritina causadas pela suplementação de vitamina A podem ser devido à mobilização dos estoques de ferro (diminuindo a ferritina) para aumentar a eritropoese (aumentando a hemoglobina). Outro estudo com gestantes da Indonésia, durante oito semanas, mostrou que um aumento significante na ferritina ocorreu somente quando a suplementação de ferro foi associada a $2,4 \mathrm{mg}$ de vitamina A. ${ }^{32}$ As diferenças entre os estudos de Tanumihardjo 32 e Muslimatun et al. 33 não eram esperadas, pois se trata de populações bem semelhantes, com valores de baseline de idade gestacional, concentração de hemoglobina e retinol sérico próximos; entretanto os valores vinicias de ferritina dos dois estudos eram diferentes, sendo os valores de Muslimatun et al. 33 inferiores aos encontrados por Tanumihardjo, $32 \mathrm{o}$ que pode, possivelmente, ser uma das razões plausíveis para a diferença encontrada.

Diferentemente dos estudos anteriormente citados, uma pesquisa conduzida com gestantes anêmicas da Indonésia não encontrou beneficio da suplementação de 2,75 mg de vitamina A, durante 60 dias, na concentração de hemoglobina; para esses autores tais resultados devem-se ao fato de as gestantes não apresentarem deficiência dessa vitamina. 34

Mulheres anêmicas, com idades entre 15 e 45 anos, foram randomizadas em grupos, recebendo ferro associado ou não a uma dose única de $60 \mathrm{mg}$ de vitamina A e zinco. Após 60 dias, observou-se que os três tratamentos (ferro, ferro+vitamina A, ferro+vitamina A+zinco) aumentaram significantemente a concentração de hemoglobina. Entretanto, o grupo que recebeu ferro+vitamina $\mathrm{A}+$ zinco apresentou maior aumento. 35

De acordo com os estudos anteriormente citados, é possível que a fortificação e suplementação com vitamina A tenha efeito favorável no metabolismo do ferro. Entretanto, devido às diferenças de duração, dose de tratamento, deficiências prévias de vitamina A e ferro e características gerais da população (como por exemplo, condições socioeconômicas), entre os estudos não é possível definir qual seria o tratamento mais adequado. Portanto, ainda são necessárias mais pesquisas para se conseguir estabelecer quais as doses e o período necessário de suplementação de vitamina A para beneficiar significantemente o estado nutricional de ferro. 


\section{Efeito da suplementação com ferro no estado nutricional de vitamina $\mathbf{A}$}

O efeito da suplementação com ferro no estado nutricional de vitamina A têm sido avaliado apenas em crianças e os resultados são contraditórios. ${ }^{36-38}$

Após três meses de suplementação com $15 \mathrm{mg}$ de ferro, crianças paquistanesas menores de dois anos demonstraram um incremento na concentração de retinol tanto no grupo que havia recebido suplementação quanto no grupo controle. Os autores atribuíram esse resultado ao aumento sazonal do consumo de alimentos fonte de vitamina $\mathrm{A}$. Entretanto, nas crianças que receberam ferro, as proteínas de fase aguda também reduziram, indicando que o possível benefício da vitamina A no estado nutricional de ferro seria a redução na frequiência de infecções, permitindo incremento da concentração plasmática de transferrina e melhora do estado nutricional do ferro. 36

Um estudo com pré-escolares mexicanos mostrou que a suplementação diária de $20 \mathrm{mg}$ de ferro esteve associada com um aumento nas concentrações de retinol e RBP (proteína de ligação do retinol), sendo que esse efeito foi maior nas crianças que tinham inicialmente deficiência de ferro e vitamina A. 37

Em crianças da Indonésia, de quatro meses de idade, foi observado que a suplementação diária de $10 \mathrm{mg}$ de ferro levou a uma redução das concentrações de retinol plasmático e, simultaneamente, ao aumento das concentrações hepáticas de vitamina A. É plausível que um dos mecanismos que poderiam explicar esses resultados seria o de que a suplementação com ferro leva a uma redistribuição do retinol do plasma para o fígado, reduzindo a quantidade de retinol circulante, podendo reduzir a disponibilidade deste para as células alvo e também induzir a um estado de deficiência funcional. Os autores explicam que a redução da vitamina A circulante pode ser devida ao aumento de sua necessidade pela aceleração da eritropoiese, e recomendam que a suplementação com ferro seja acompanhada de medidas que melhorem o estado nutricional da vitamina A. 38

\section{Possíveis mecanismos da interação entre ferro e vitamina A}

Embora existam fortes evidências da associação entre ferro e vitamina $\mathrm{A}$, os mecanismos para explicá-la ainda são obscuros. A anemia ferropriva é o último estágio da deficiência de ferro. O primeiro estágio, chamado de depleção dos estoques, é caracterizado pela progressiva redução na quantidade desse mineral estocado no fígado. Nesse caso, a mobilização hepática do ferro pode não ocorrer e consequentemente quantidades insuficientes do mineral seriam transportadas através da transferrina, levando a uma redução progressiva na saturação da mesma, caracterizando assim o segundo estágio. Quando a saturação da transferrina chega a um limite crítico, os precursores do eritrócito, os quais necessitam continuamente de ferro para a formação da hemoglobina, podem ter seus suprimentos de ferro insuficientes, ocorrendo, portanto um declínio dos níveis circulantes de ferro; esse é o estágio mais avançado da deficiência, chamado de anemia ferropriva. O diagnóstico nesse estágio é caracterizado pela redução da hemoglobina e dos níveis de ferro sérico, aumento da capacidade total de ligação do ferro, baixo volume corpuscular médio e redução da concentração de ferritina. ${ }^{1} \mathrm{Na}$ anemia causada pela deficiência de vitamina A, diferente da anemia ferropriva, os níveis de ferritina são normais. 14

Há evidências de que a associação entre vitamina A e ferro deva-se ao fato de a vitamina A beneficiar a eritropoese, prevenir infecções ou melhorar a absorção de ferro. 12,15,29,39-42

\section{Eritropoese}

A revisão de Hodges et al.,39 baseada em estudos relacionando a vitamina $\mathrm{A}$ e a hematopoiese, suporta o conceito que a vitamina A é essencial para hematopoese normal, pois existe um acúmulo de ferro no fígado de ratos deficientes em vitamina A, sugerindo uma alteração no uso e/ou mobilização dos estoques de ferro. Assim, na anemia causada pela deficiência de vitamina A ocorreriam baixas concentrações de ferro sérico e elevados níveis de ferro estocado principalmente, no fígado. Mejía e Arroyave 26 e Muslimatun et al. ${ }^{33}$ sugerem uma ação da vitamina A na mobilização dos estoques de ferro, pois a fortificação com essa vitamina aumentou a biodisponibilidade do ferro sérico para hematopoiese devido à depleção dos estoques.

É importante ressaltar que existe uma relação inversa entre os estoques de vitamina A e ferro no fígado, fato que explica o papel da vitamina A na prevenção da anemia, pois, na ausência dessa vitamina, o ferro no fígado ficaria aprisionado e a anemia ocorreria apesar dos elevados estoques de ferro naquele órgão. ${ }^{43}$ Posteriormente, confirmou-se que na anemia causada pela deficiência de vitamina A os níveis de ferritina são normais, apesar da baixa concentração de ferro sérico e hemoglobina. 14

Outros estudos também mostram as relações 
entre vitamina A e hematopoese: o benefício da suplementação de vitamina A na hematopoese ${ }^{19,28}$ e a deficiência de vitamina A diminuindo a síntese de transferrina e assim reduzindo o transporte de ferro para a medula óssea. ${ }^{26,28}$ Postulou-se que o principal efeito da vitamina A é manter os níveis de ferro adequados no plasma, para suprir os diferentes tecidos, incluindo a medula óssea, com adequadas quantidades de ferro. 27

Bloem 14 acrescenta que a suplementação de vitamina A sempre resulta em um incremento de ferro sérico, mas nem sempre nos níveis de hemoglobina; segundo o autor essa observação suporta a hipótese de que o efeito primário da suplementação de vitamina A está na elevação do ferro sérico e que a mudança pode favorecer a hematopoese através de um aumento da concentração de hemoglobina.

\section{A anemia da infecção}

Acredita-se que a ligação entre ferro e vitamina A está no fato de ambos serem transportados por proteínas negativas de fase aguda, RBP (proteína de ligação do retinol) e transferrina, as quais têm sua síntese deprimida na presença de infecção. Assim, com baixas concentrações de retinol, a infecção seria mais facilmente instalada e haveria acúmulo de ferro no fígado, causando anemia. 12 Segundo Bloem, 14 a chave da hipótese apresentada acima está no fato de a vitamina A oferecer propriedades imunes. Assim, com a suplementação dessa vitamina poder-se-ia esperar que a infecção fosse suprimida e que ocorresse estímulo na síntese de transferrina e RBP, liberando o ferro e o retinol do fígado.

Northrop-Clewes et al. 36 sugeriram que a administração de vitamina $\mathrm{A}$ reduz as frequiências de infecção e que a mudança da resposta da fase aguda permite um aumento da concentração plamática de transferrina e, consequentemente, melhora do estado nutricional de ferro.

Embora a hipótese de que a deficiência de vitamina A contribui para anemia através da depressão da imunidade para infecções seja razoável, Semba e Bloem 42 consideram que ainda existem poucos dados que suportam essa hipótese.

\section{Melhora na absorção do ferro dos alimentos}

Tem sido sugerida uma possível melhora da absorção de ferro na presença da vitamina A.44 Garcia-Casal et al.41 mostraram em estudos com isótopos que baixas doses de vitamina $\mathrm{A}$ ou $\beta$ - caroteno podem dobrar a absorção de ferro nãoheme dos cereais em adultos anêmicos, devido, provavelmente, à prevenção dos efeitos inibitórios de fitatos na absorção de ferro. Entretanto, Walczyk et al. 45 não encontraram alteração na absorção do ferro por influência da vitamina $\mathrm{A}$, acreditando ser possível que a vitamina A melhore a absorção do ferro apenas em indivíduos com deficiência dessa vitamina. Entretanto, os autores sugerem que mais estudos sejam realizados com intuito de prover estas respostas.

Acredita-se que a melhora no metabolismo do ferro, quando vitamina A é suplementada, deve-se, não a melhora da absorção do ferro, mas à mobilização do estoque de ferro disponível e aumento da utilização desse ferro para formação de hemoglobina, reduzindo os estoques de ferro e então desencadeando aumento na absorção desse mineral. 28

Como se pode observar, os estudos que avaliam a influência da vitamina A na absorção no ferro ainda apresentam resultados contraditórios. Segundo Davidsson et al.,46 essas informações contraditórias sobre a influência da vitamina A na melhora da absorção de ferro indicam a complexidade das interações entre vitamina A e metabolismo do ferro, e sugerem que mais estudos sejam realizados com intuito de esclarecer estas dúvidas.

\section{Conclusões}

Os estudos descritos nesta revisão demonstram que nos grupos de crianças, gestantes e adolescentes há fortes evidências que apóiam a hipótese de uma correlação entre vitamina A e ferro e, mesmo que essas associações não tenham sido fortes, encontrouse que a suplementação com vitamina A melhora o estado nutricional de ferro, com reduções significantes nas prevalências de anemia.

Assim, é possível admitir que a fortificação de alimentos e a suplementação combinada com vitamina A possam ter papel crucial no controle da anemia, principalmente em países em desenvolvimento, onde essa enfermidade é mais prevalente. Portanto, sugere-se que programas que tenham o objetivo de reduzir a anemia devam combinar a suplementação de ferro com vitamina A para melhorar a eficácia dos programas de prevenção e controle. Para tal, são necessárias mais experiências clínicas feitas com intuito de avaliar qual o esquema posológico de intervenção com vitamina A que mostre maior impacto no incremento das concentrações de hemoglobina em populações onde a anemia nutricional ferropriva tem distribuição endêmica. 


\section{Referências}

1. FAO (Food and Agriculture Organization) WHO (World Health Organization). Iron. In: FAO/WHO. Human vitamin and mineral requeriments. Report of a Joint FAO/WHO Expert Consultation. Bangkok; 2001.p. 195-221.

2. Olivares M, Walter T, Cook JD, Hertrampf E, Pizarro F. Usefulness of serum transferrin receptor and serum ferritin in diagnosis of iron deficiency in infancy. Am J Clin Nutr. 2000; 72: 1191-5.

3. Neuman NA, Tanaka OY, Szarfarc SC, Guimarães PRV, Victora CG. Prevalência e fatores de risco para anemia no Sul do Brasil. Rev Saúde Pública. 2000; 34: 56-63.

4. Allen LH. Anemia and iron deficiency: effects on pregnancy outcome. Am J Clin Nutr. 2000; 71 (Suppl):S1280S45.

5. Nelson M. Anemia in adolescent girls. Proc Nutr Soc. 1996; 55: 359-67.

6. Haas JD, Brownlie T. Iron deficiency and reduced work capacity: a critical review of the research to determine a causal relationship. J Nutr 2001; 131: (25-2): S676-88S.

7. FAO (Food and Agriculture Organization)/ WHO (World Health Organization). Vitamin A. In: FAO/WHO. Human vitamin and mineral requeriments. Report of a Joint FAO/WHO Expert Consultation. Bangkok; 2001. p. 87-107

8. Bates CJ. Vitamin A. Lancet. 1995; 345: 31-5.

9. Albalak R, Ramakrishnan U, Stein AD, Haar FV, Haber MJ, Schroeder D, Martorell R. Co-occurrence of nutrition problems in Honduran children. J Nutr. 2000; 130: 2271-3.

10. Palafox NA, Gamble MV, Dancheck B, Ricks MO, Briand K, Semba RD. Vitamin A deficiency, iron deficiency, and anemia among preschool children in the Republic of the Marshall Islands. Nutrition. 2003; 19: 405-8.

11. Dijkhuizen MA, Wieringa FT, West CE, Muherdiyantiningsih, Muhilal. Concurrent micronutrient deficiencies in lactanting mothers and their infants in Indonesia. Am J Clin Nutr. 2001; 73: 786-91.

12. Thurnham DI. Vitamin A, iron and haemopoiesis. Lancet. 1993; 342: 1312-3

13. Semba RD. The role of vitamin A and related retinoids in immune function. Nutr Rev. 1998; 56: 38S-48S.

14. Bloem MW. Interdependence of vitamin A and iron: an important association for programmes of anaemia control. Proc Nutr Soc. 1995; 54: 501-8.

15. Bloem MW, Wedel M, Egger RJ, Speek AJ, Scrijver J, Saowakontha S, Schreurs WHP. Iron metabolism and vitamin A deficiency in children in Northeast Thailand. Am J Clin Nutr. 1989; 50: 332-8.

16. Wold-Gebriel BZ, West CE, Gebru H, Tadesse AS, Fisseha T, Gabre P, Aboye C, Ayana G, Hautvast JGA. Interrelation between vitamin $\mathrm{A}$, iodine and iron status in schoolchildren in Shoa Region, Central Ethiopia. Br J Nutr. 1993; 70: $593-$ 607.

17. Allen LH, Rosado JL, Casterline JE, Lópes P, Munõz E, Gracia OP, Martinez H. Lack of hemoglobin response to iron supplementation in anemic Mexican preschoolers with multiple micronutrient deficiencies. Am J Clin Nutr 2000; 71: $1485-94$.
18. Magalhães P, Andréa R, Ramalho CC. Deficiência de ferro e de vitamina A: avaliação nutricional de pré-escolares de Viçosa (MG/Brasil). Nutrire: Rev Soc Bras Alim Nutr. 2001; 21: 41-56.

19. Willows ND, Gray-Donald K. Serum retinol is associated with hemoglobin concentration in infants who are not vitamin A deficient. Nutr Res. 2003; 23: 891-900.

20. Hinderaker SG, Olsen BE, Lie RT, Bergsjo PB, Gasheka P, Bondevik GT, Ulvik R, Kvale G. Anemia in pregnancy in rural Tanzania: associations with micronutrients status and infections. Eur J Clin Nutr. 2002; 56: 192-9.

21. Suharno D, West CE, Muhilal, Logman MHGM, Waart FG, Karyadi D, Hautvast GAJ. Cross-sectional study on the iron and vitamin A status of pregnant women in West Java, Indonesia. Am J Clin Nutr. 1992; 56: 988-93.

22. Ahmed F, Khan MR, Karim R, Taj S, Hyderi T, Faruque MO, Margetts BM, Jackson AA. Serum retinol and biochemical measures of iron status in adolescent schoolgirls in urban Bangladesh. Eur J Clin Nutr. 1996; 50: 34651

23. Fazio-Tirozzo G, Brabin L, Brabin B, Agbaje O, Harper G, Broadhead R. A community based study of vitamin A and vitamin E status of adolescent girls living in the Shire Valley, Southern Malawi. Eur J Clin Nutr. 1998; 52: 63742

24. Ahmed F, Khan MR, Jackskon AA. Concomitant supplemental vitamin A enhances the response to weekly supplemental iron and folic acid in anemic teenagers in urban Bangladesh. Am J Clin Nutr. 2001; 74: 108-15.

25. Ettyang GA, Lichtenbelt WDM, Oloo A, Saris WHM. Serum retinol, iron status and body composition of lactanting women in Nandi, Kenya. Ann Nutr Metab. 2003; 47: $276-83$

26. Mejía LA, Arroyave G. The effect of vitamin A fortification of sugar on iron metabolism in preschool children in Guatemala. Am J Clin Nutr. 1982; 36: 87-93.

27. Mejía LA, Chew F. Hematological effect of supplementing anemic children with vitamin A alone and in combination with iron. Am J Clin Nutr. 1988; 48: 595-600.

28. Bloem MW, Wedel M, Agtmaal EJV, Speek AJ, Saowakontha S, Schreurs WHP. Vitamin A intervention: short-term effects of a single, oral, massive dose on iron metabolism. Am J Clin Nutr. 1990; 51: 76-9.

29. Mwanri L, Worsley A, Ryan P, Masika J. Supplemental vitamin A improves anemia and growth in anemic school children in Tanzania. J Nutr. 2000; 130: 2691-6.

30. Suharno D, West CE, Muhilal, Karyadi D, Hautvast JGAJ. Supplementation with vitamin A and iron for nutritional anaemia in pregnant women in West Java, Indonesia. Lancet. 1993; 342: 1325-8.

31. Panth M, Shatrugna V, Yasodhara P, Sivakumar B. Effect of vitamin A supplementation on haemoglobin and vitamin A levels during pregnancy. Br J Nutr. 1990; 64: 351-8.

32. Tanumihardjo SA. Vitamin A and iron status are improved by vitamin A and iron supplementation in pregnant Indonesian women. J Nutr. 2002; 132: 1909-12. 
33. Muslimatun S, Schmidt MK, Schultink W, West CE, Hautvast JGAJ, Gross R, Muhilal. Weekly supplementation with iron and vitamin A during pregnancy increases hemoglobin concentration but decreases serum ferritin concentration in Indonesian pregnant women. J Nutr. 2001; 131: $85-90$

34. Suprapto B, Widardo, Suhanantyo. Effect of low-dosage vitamin A and riboflavin on iron-folate supplementation in anaemic pregnant women. Asia Pacific J Clin Nutr. 2002; 11: 263-7.

35. Kolsteren P, Rahman SR, Hilderbrand K, Diniz A. Treatment for iron deficiency anaemia with a combined supplementation of iron, vitamin A and zinc in women of Dinajpur, Bangladesh. Eur J Clin Nutr. 1999; 53: 102-6.

36. Northrop-Clewes CA, Paracha PI, Mcloone UJ, Thurnham DI. Effect of improved vitamin A status on response to iron supplementation in Pakistani infants. Am J Clin Nutr. 1996; 64: 694-9.

37. Muñoz EC, Rosado JL, López P, Furr HC, Allen LH. Iron and zinc supplementation improves indicators of vitamin A status of Mexican preschoolers. Am J Clin Nutr. 2000; 71: 789-94.

38. Wieringa TF, Dijkhuizen AM, West EC, Thurnham ID, Muhilal, Van Der Meer MWJ. Redistribution of vitamin A after iron supplementation in Indonesian infants. Am J Clin Nutr. 2003; 77: 651-7.

39. Hodges RE, Sauberlich HE, Canham JE, Wallace DL Rucker RB, Mejia LA, Mohanram M. Hematopoietic studies in vitamin A deficiency. Am J Clin Nutr. 1978; 31: $776-885$.

Recebido em 1 de outubro de 2005

Versão final apresentada em 20 de dezembro de 2006

Aprovado em 8 de janeiro de 2007.
40. West CE. Strategies to control nutritional anemia. Am J Clin Nutr. 1996; 64: 789-90.

41. Garcia-Casal MN, Layrisse M, Solano L, Barón MA, Arguello F, Llovera D, Ramirez J, Leets I, Topper E. Vitamin $A$ and $\beta$-caroteno can improve nonheme iron absorption from rice, wheat and corn by humans. J Nutr. 1998; 128: 646-50.

42. Semba RD, Bloem MW. The anemia of vitamin A deficiency: epidemiology and pathogenesis. Eur J Clin Nutr. 2002; 56: 271-81

43. Staab DB, Hodges RE, Metcalf WK, Smith JL. Relationship between vitamin A and iron in the liver. J Nutr. 1984; 114: 840-4.

44. Garcia-Casal MN, Layrisse M. Food iron absorption: role of vitamin A. Arch Latinoam Nutr. 1998; 48: 191-6.

45. Walczyk T, Davidsson L, Rossander-Hulthen L, Halberg L, Hurrell RF. No enhancing effect of vitamin A on iron absorption in humans. Am J Clin Nutr. 2003; 77: 144-9.

46. Davidsson L, Adou P, Zeder C, Walczyk T, Hurrell R. The effect of retinyl palmitate added to iron-fortified maize porridge on erythrocyte incorporation of iron in African children with vitamin A deficiency. Br J Clin Nutr. 2003; 90: $337-43$ 